\title{
Landscape genetics as a tool for conservation planning: predicting the effects of landscape change on gene flow
}

\author{
Maarten J. van Strien, ${ }^{1,2,4}$ Daniela Keller, ${ }^{2,3}$ Rolf Holderegger,,${ }^{2,3}$ Jaboury Ghazoul, ${ }^{3}$ Felix Kienast, ${ }^{2,3}$ \\ AND JANINE BOLLIGER ${ }^{2,3}$ \\ ${ }^{1}$ Planning of Landscape and Urban Systems, ETH Zurich, Wolfgang-Pauli-Str. 15, CH-8093 Zurich, Switzerland \\ ${ }^{2}$ WSL Swiss Federal Research Institute, Zürcherstrasse 111, CH-8903 Birmensdorf, Switzerland \\ ${ }^{3}$ Department of Environmental Systems Science, ETH Zurich, Universitätsstrasse 16, CH-8092 Zurich, Switzerland
}

\begin{abstract}
For conservation managers, it is important to know whether landscape changes lead to increasing or decreasing gene flow. Although the discipline of landscape genetics assesses the influence of landscape elements on gene flow, no studies have yet used landscapegenetic models to predict gene flow resulting from landscape change. A species that has already been severely affected by landscape change is the large marsh grasshopper (Stethophyma grossum), which inhabits moist areas in fragmented agricultural landscapes in Switzerland. From transects drawn between all population pairs within maximum dispersal distance $(<3 \mathrm{~km})$, we calculated several measures of landscape composition as well as some measures of habitat configuration. Additionally, a complete sampling of all populations in our study area allowed incorporating measures of population topology. These measures together with the landscape metrics formed the predictor variables in linear models with gene flow as response variable ( $F_{\mathrm{ST}}$ and mean pairwise assignment probability). With a modified leave-oneout cross-validation approach, we selected the model with the highest predictive accuracy. With this model, we predicted gene flow under several landscape-change scenarios, which simulated construction, rezoning or restoration projects, and the establishment of a new population. For some landscape-change scenarios, significant increase or decrease in gene flow was predicted, while for others little change was forecast. Furthermore, we found that the measures of population topology strongly increase model fit in landscape genetic analysis. This study demonstrates the use of predictive landscape-genetic models in conservation and landscape planning.
\end{abstract}

Key words: agricultural landscapes; conservation management; forecasting; gene flow; planning tool; Stethophyma grossum.

\section{INTRODUCTION}

Habitat loss and fragmentation resulting in small and less-connected populations have been identified as threats to the long-term survival of species (Lande 1998). Reduced connectivity and gene flow can decrease the resilience, adaptability, fitness, and fertility of populations, especially if populations are small (Frankham 2006). Whereas anthropogenic causes of habitat loss and fragmentation can be avoided or reduced to some extent within designated nature protection areas, these processes are difficult to mitigate in humandominated landscapes such as agricultural landscapes (Bennett et al. 2006). In such landscapes, it is important to determine whether human-induced changes to the landscape will result in significant changes to gene flow in species inhabiting these landscapes.

Determining the influence of landscape configuration and composition on gene flow is the focus of the discipline of landscape genetics (Manel et al. 2003,

Manuscript received 7 March 2013; revised 5 June 2013; accepted 26 June 2013. Corresponding Editor: A. K. Brody.

${ }^{4}$ E-mail: maarten@vstrien.nl
Holderegger and Wagner 2008). Most landscape-genetic studies are exploratory; their goal is to identify landscape elements that have either an inhibiting or facilitating effect on gene flow (Storfer et al. 2010). However, many of the models developed in landscapegenetic studies could, in principle, also be used for predictive purposes, i.e., to forecast the effects of landscape changes on genetic patterns. Storfer et al. (2007:138) identified such predictive studies as “... an important future application of landscape genetics" and point out that "this type of study may allow managers to choose a management alternative that minimizes impacts on the focal species while allowing some development to take place." Although the importance of predictive landscape-genetic studies for conservation management has been recognized by several authors (Storfer et al. 2007, Balkenhol et al. 2009a, Spear et al. 2010), no studies have yet experimented in this direction (Spear et al. 2010). Therefore, the goal of this study is to demonstrate the application of landscape-genetic models to predict the effects of landscape change on gene flow among populations. 
A species that inhabits agricultural landscapes and that has been negatively affected by habitat loss and fragmentation is the large marsh grasshopper (Stethophyma grossum). The eggs of this species only develop at high soil moisture levels (Ingrisch 1983), which makes $S$. grossum reproductively bound to wet areas like wetlands, marshes, wet meadows, and swampy areas alongside streams, lakes, and rivers (Baur et al. 2006). Considering that $>90 \%$ of Swiss wetlands and floodplains have disappeared since 1800 (SAEFL 1998), it is not surprising that $S$. grossum is red-listed as vulnerable in Switzerland (Monnerat et al. 2007). Ecological studies have already identified structural landscape elements, such as rows of tall trees (Sorens 1996, Bonsel and Sonneck 2011) and high-banked roads (Marzelli 1995), as potential barriers to $S$. grossum movement. Therefore, we focus on this species to determine what changes in gene flow can be foreseen after landscape alterations.

In a fragmented agricultural area of Switzerland, we performed a complete sampling of all $S$. grossum populations. Individuals were genotyped, and measures of genetic distance between populations were determined (Keller et al. 2013a). In a four-step landscape-genetic approach, (1) we created a set of potential predictor variables by determining the proportion of several landscape elements within transects drawn between pairs of populations (Emaresi et al. 2011, Keller et al. 2012b). In addition to these measures of landscape composition, we calculated several more complex measures of habitat configuration from these transects (Schumaker 1996, McGarigal et al. 2012). The effect of the spatial arrangement of populations (i.e., population topology) on gene flow has been identified as an important aspect in landscape genetics (Keller et al. 2013a). Therefore, by determining the number of populations surrounding a population pair and the average distance to these populations, we also introduce two predictor variables to quantify population topology in landscape-genetic models. From the complete set of predictor variables, (2) we selected a subset that had the best predictive accuracy based on a cross-validation approach modified to accommodate for the nonindependence of response and predictor variables derived from distance matrices as commonly used in landscape genetics. Subsequently, (3) we described hypothetical landscape-change scenarios. We chose scenarios in which a residential area is constructed, agricultural land is reforested, forest is converted to agricultural land, or a new population is established between existing populations. We quantified the influence of these scenarios on the selected predictor variables. With these new predictor values (4), we predicted future gene flow values and determined whether the corresponding change in gene flow was significant.

\section{Methods}

Study area and species

The study area $\left(\sim 180 \mathrm{~km}^{2}\right)$ was located around the town of Langenthal $\left(47^{\circ} 12^{\prime} 50^{\prime \prime} \mathrm{N}, 7^{\circ} 47^{\prime} 05^{\prime \prime} \mathrm{E}\right)$ on the
Swiss plateau. The landscape primarily consists of intensive agriculture intermixed with forest patches and settlements (Fig. 1). Three parallel streams flow through the area that empty into the major river Aare. The stream basins create suitable habitats for populations of S. grossum (Linnaeus, 1758; Acrididae).

Low levels of genetic differentiation have been detected between the populations of $S$. grossum in this study area (Keller et al. 2013a), indicating that there was regular gene flow between populations. Mark-releaserecapture studies have recorded a maximum dispersal distance of $624 \mathrm{~m}$ for S. grossum (Malkus 1997, Bonsel and Sonneck 2011). Furthermore, single individuals have been observed $1500 \mathrm{~m}$ from nearest populations (Griffioen 1996). However, recordings from sighting studies can easily underestimate true dispersal distances (Franzén and Nilsson 2007, Hassall and Thompson 2012). Especially if species exhibit partial migration (i.e., most individuals are sedentary and only a few disperse over longer distances [Chapman et al. 2011]), the few long-distance migrants can easily be overlooked in sighting studies. Judging from the frequency of movement distribution presented in Bonsel and Sonneck (2011), partial migration is present in S. grossum. Therefore, we estimated the maximum dispersal distance of $S$. grossum to be $3000 \mathrm{~m}$, which is in accordance with the genetic results of Keller et al. (2013a).

\section{Genetic data set}

The genetic data set used here has been described in Keller et al. $(2013 a, b)$. In brief, we performed a complete sampling of all populations of $S$. grossum in the study area in July and August 2010. All potentially moist areas (i.e., river or stream banks, valley bottoms, lake shores, ditches and swamps) were checked for the presence of $S$. grossum individuals. Samples were taken only if populations were located at least $350 \mathrm{~m}$ from the nearest known population. From each population, up to 30 individuals were sampled by removing the tibia and tarsus of one mid-leg and storing those in $100 \%$ ethanol. In the genetic analysis, seven polymorphic microsatellite markers (Sgr10, Sgr13, Sgr15, Sgr19, Sgr38, Sgr40, and Sgr45 [Keller et al. 2012a]) were amplified, analyzed on an ABI 3730xl sequencer (Applied Biosystems; Life Technologies, Carlsbad, California, USA) and scored with GENEMAPPER 3.7 (Applied Biosystems). These seven markers were in Hardy-Weinberg equilibrium, had low null allele frequencies, and also a low genotyping error rate averaging $4.7 \%$ (Keller et al. 2013a). Such an error rate is comparable to other genetic analyses using microsatellites and acceptable to calculate genetic differentiation between populations based on allele frequencies (Selkoe and Toonen 2006).

For further analysis, we selected only those populations from which five or more individuals had been sampled, and also removed two remote populations that were outside the complete-sampling area (Fig. 1 and Keller et al. 2013a). This resulted in a total of 37 


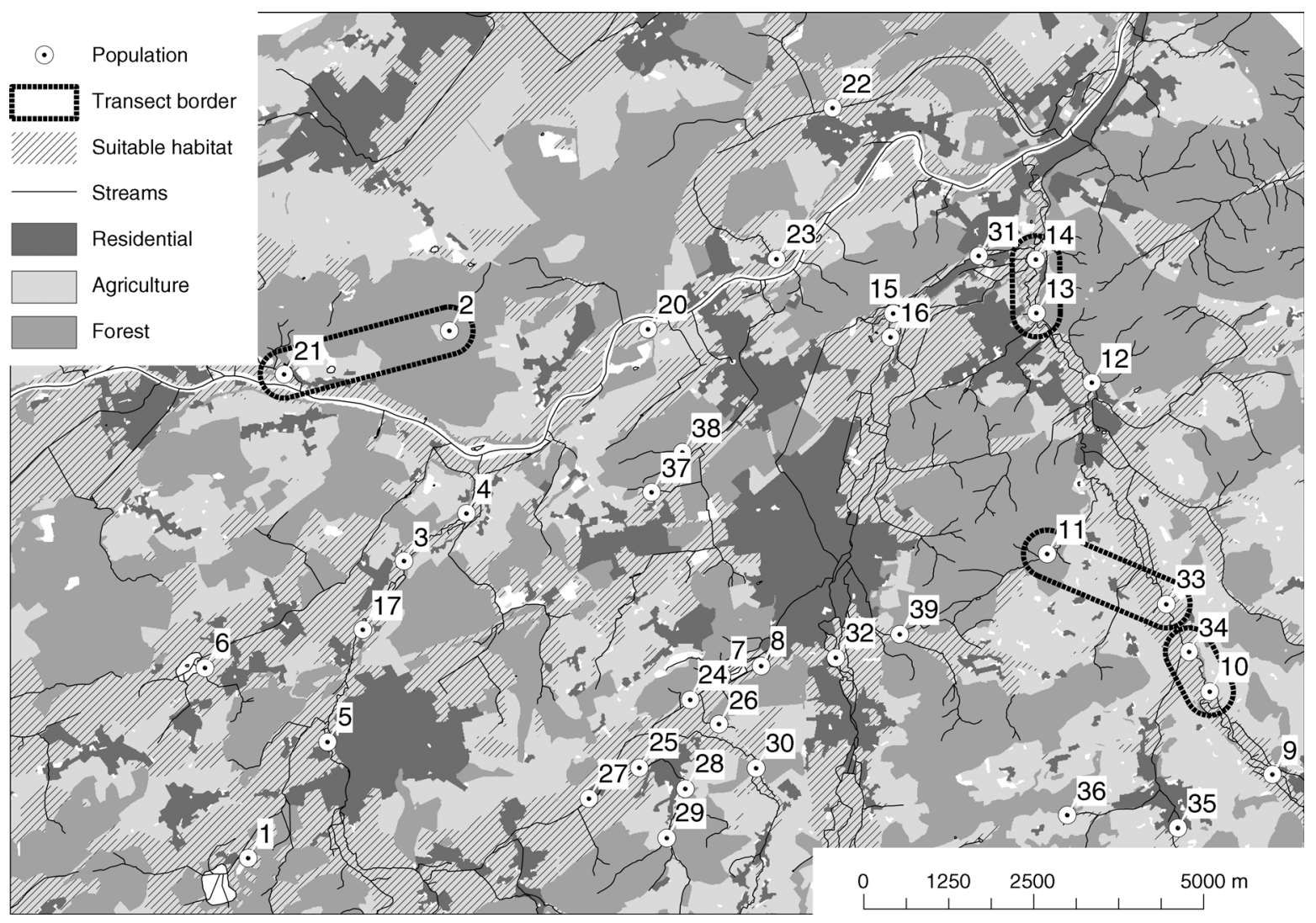

FIG. 1. Map of the study area in Switzerland, in which all populations of Stethophyma grossum were sampled. Also shown is the spatial distribution of the landscape elements that were selected as predictor variables in regression modeling. Habitat (hatched area) was mapped by selecting all flat locations close to water $(<500 \mathrm{~m})$ and on agricultural land. A unique identification number was given to each of the 37 populations. For the two population pairs that were genetically most isolated (pairs 13-14 and 10-34) and least isolated (pairs 11-33 and 2-21) from each other, the border of the respective transect (dashed line) from which landscape metrics were calculated is indicated. These transects were also used to predict changes in gene flow caused by landscape change (Fig. 5).

populations. As measures of gene flow between populations, we used both pairwise $F_{\mathrm{ST}}$ values (FSTAT 2.9.3.2; Goudet 1995) and pairwise mean assignment probabilities (MAP; Keller et al. 2013a,b). The latter is derived from assignment probabilities calculated with GENECLASS 2.0.h (Piry et al. 2004). MAP between populations $\mathrm{A}$ and $\mathrm{B}$ is the mean of the assignment probabilities of individuals in population A to population $\mathrm{B}$, and vice versa. Thus, the higher MAP is, the more gene flow is expected. Gene flow between any two populations beyond the maximum dispersal distance of S. grossum (i.e., $3 \mathrm{~km}$; see Abstract) is probably directed over several generations via intermediate populations (e.g., McRae 2006). Thus, the landscape directly between these two populations may not give a good representation of the landscape encountered by the majority of "indirect migrants" between the two populations. Therefore, we only performed our landscape-genetic analysis on population pairs that were within maximum dispersal distance (i.e., $\leq 3 \mathrm{~km}$; see Abstract), which resulted in a data set of 97 population pairs.

\section{Predictor variables}

In landscape genetics, estimates of gene flow are considered a function of interpopulation distance and the intervening landscape. Several methods are currently used to quantify the landscape between populations (Spear et al. 2010). Recently, several studies have used straight-line interpopulation transects of a certain width, from which several landscape predictor variables are calculated (Pavlacky et al. 2009, Angelone et al. 2011, Emaresi et al. 2011, Keller et al. 2012b). In this transect method, a separate predictor variable is calculated for each landscape element (Emaresi et al. 2011), which is a desirable property if the model is to be used to predict the effect of changes of specific landscape elements.

We experimented with a range of transect widths (i.e., $100,200,400,700$, and $1000 \mathrm{~m}$ ), from which we calculated several landscape predictor variables. To account for isolation-by-distance (Jenkins et al. 2010), we used the natural log-transformed length of the transect $(\ln [\mathrm{DISTANCE})$. From land cover grids $(10$ $\times 10 \mathrm{~m}$ spatial resolution) derived from 1:25000 vectorized land cover maps of Switzerland (Vector25 

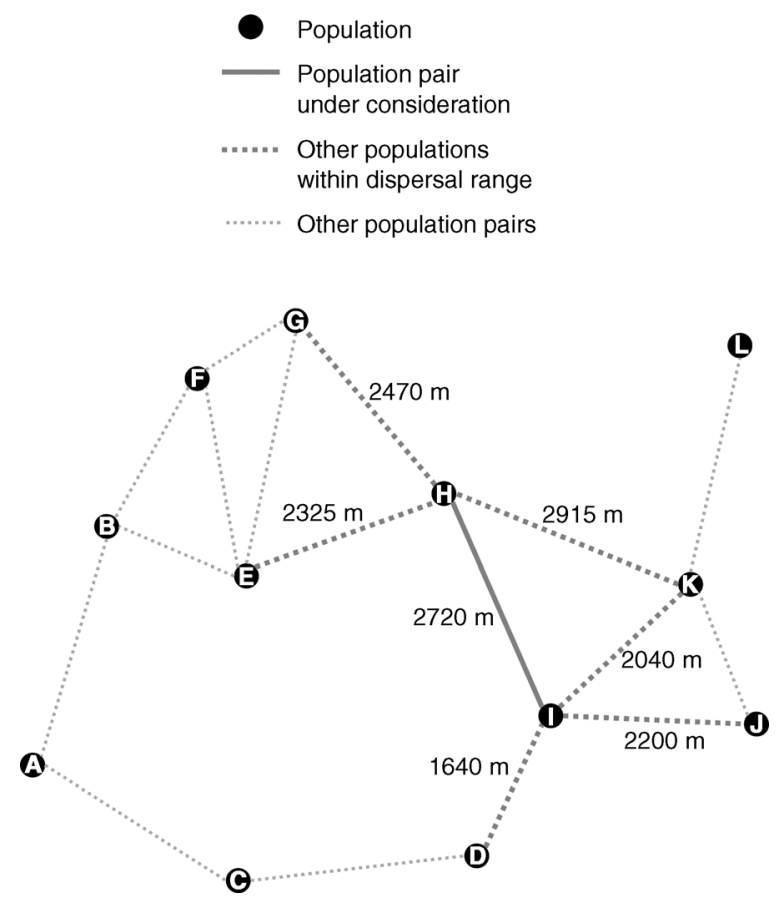

FIG. 2. Demonstration of the calculation of population topology predictor variables used in this study. In this hypothetical population network, all population pairs within the maximum dispersal distance (i.e., $\leq 3 \mathrm{~km}$ ) of one another are linked. The number of links (NL) from population $\mathrm{H}$ to other populations is 4 (i.e., to populations E, G, I, and K), and the mean distance of these links (MDL) is $(2325+2470+2720$ $+2915) / 4=2608 \mathrm{~m}$. Similarly, for population I, NL is 4 (i.e., to populations $\mathrm{D}, \mathrm{H}, \mathrm{K}$, and $\mathrm{J})$, and MDL is $(1640+2720+2040$ $+2200) / 4=2150 \mathrm{~m}$. Mean NL $(\mathrm{MEAN}-\mathrm{NL}=(4+4) / 2=4)$ and mean MDL $($ MEAN-MDL $=(2608+2150) / 2=2379 \mathrm{~m})$ are then assigned to population pair $\mathrm{H}-\mathrm{I}$ as population topology predictor variables.

2011; Swisstopo; DV033594; available online), ${ }^{5}$ we then calculated the proportion of landscape elements in the transects as measures of landscape composition (Pavlacky et al. 2009, Angelone et al. 2011, Emaresi et al. 2011, Keller et al. 2012b). As potential barriers to gene flow in $S$. grossum, we considered the proportion of residential area (RESIDENTIAL; $\mathrm{m}^{2} / \mathrm{m}^{2}$ ) and the proportion of forest (FOREST; $\mathrm{m}^{2} / \mathrm{m}^{2}$; Fig. 1). As potential facilitators to gene flow, we selected the proportion of agricultural land (AGRICULTURE; $\mathrm{m}^{2} / \mathrm{m}^{2}$ ), the proportion of streams (STREAMS; $\mathrm{m}^{2} /$ $\mathrm{m}^{2}$ ) and the proportion of suitable habitat (HABITAT; $\mathrm{m}^{2} / \mathrm{m}^{2} ;$ Fig. 1). Suitable habitat was mapped in the study area by selecting all areas that were close to open water $(\leq 500 \mathrm{~m})$, in open agricultural land and relatively flat with little runoff (i.e., all areas differing not more than $20 \mathrm{~m}$ in elevation compared to the lowest point in a 500 $\mathrm{m}$ radius [Keller et al. 2013a]). Since the proportion of agricultural land was strongly correlated to the propor-

\footnotetext{
${ }^{5}$ http://www.swisstopo.admin.ch/internet/swisstopo/en/ home/apps/geodata_portal.html
}

tion of forest (Spearman $\rho<-0.68$ ) in all transect widths, we decided to remove the latter from further analysis.

Apart from the above landscape predictor variables that quantify landscape composition, we also calculated measures of habitat configuration from the transects, as they may give a better representation of the landscape that a dispersing individual encounters (e.g., Schumaker 1996). We focused on class-level statistics (McGarigal et al. 2012), in which the landscape class under consideration was the mapped suitable habitat. Binary maps of suitable habitat and matrix were created for each transect and used as input for FRAGSTATS 4.0 (McGarigal et al. 2012). As many landscape metrics are highly correlated and conceptually similar (Riitters et al. 1995), we selected two ecologically meaningful indices that are supposed to be insensitive to transect size. The first landscape index was the patch cohesion index (COHESION), which was calculated from the perimeter and area of the habitat patches in the transect and the total area of the transect (McGarigal et al. 2012). This index measures the connectedness of habitat patches and has proved to correlate well to estimates of dispersal (Schumaker 1996). Second, we calculated the aggregation index (AI), which measures the aggregation or clumpiness of a landscape class (He et al. 2000). This index was calculated from the number of adjoining habitat pixels in the transect and the theoretical maximum number of adjoining habitat pixels (McGarigal et al. 2012). We found COHESION and AI to be highly correlated with HABITAT (Spearman $\rho>0.69$ ) for some, but not all, transect widths, so we retained these three predictor variables in the final list of landscape predictor variables. All spatial analyses were performed in ARCGIS 9.3 (Esri, Redlands, California, USA), unless otherwise specified.

Recently, several studies have emphasized the need to take population topology (i.e., the arrangement of populations in the landscape) into account in landscape-genetic analysis (McRae 2006, Dyer et al. 2010, Keller et al. 2013a). Gene flow between two populations can, over several generations, take place via intermediate populations. The establishment or disappearance of intermediate populations may thus influence gene flow between populations. Therefore, we experimented with two predictor variables that aim to quantify population topology around a given population pair. For each population, we determined the number of populations that were within maximum dispersal distance (number of links: NL) and the mean distance to these populations (mean distances of links: MDL). We then determined the mean of both measures per population pair (i.e., MEAN-NL and MEAN-MDL, respectively; Fig. 2). We expected that the more populations surround a population pair and/or the closer these surrounding populations are to either of the two populations, the higher is gene flow between the two populations. To determine whether these two predictor variables have any predic- 
tive power in landscape-genetic analysis, we tested the predictive ability of models with and without these variables. We checked that both MEAN-NL and MEAN-MDL were never in the final model, as these predictor variables were highly correlated (Spearman $\rho$ $=-0.74)$.

\section{Predictive models and cross-validation}

We used multiple linear regression to correlate the response variable (i.e., either $F_{\mathrm{ST}}$ or MAP) to the suite of predictor variables (i.e., $\ln$ (DISTANCE), STREAMS, HABITAT, RESIDENTIAL, AGRICULTURE, AI, COHESION, MEAN-NL, and MEAN-MDL). A primary goal of the statistical analysis in this study was to select the subset of predictor variables that had the highest predictive accuracy. Two approaches that have been advocated for variable selection are information criterion (e.g., AIC) and cross-validation (Burnham and Anderson 2002). It has been shown that these approaches (i.e., AIC and leave-one-out cross-validation) are asymptotically equivalent (Stone 1977, Shao 1993). We chose to use leave-one-out cross-validation (LOOCV) for variable selection, as this method is commonly used to estimate prediction accuracy (Verbyla and Litvaitis 1989, Molinaro et al. 2005), and after making some slight adaptations, allowed us to verify the predictive model on pairwise $F_{\mathrm{ST}}$ or MAP values that are "independent" (i.e., the model is validated on $F_{\mathrm{ST}}$ or MAP values that are not used to fit the model). With normal LOOCV an "independent validation" of a model is simulated by fitting a regression model to a calibration (or estimation or training) set, which consists of the whole data set excluding one observation (i.e., the validation, evaluation, or testing set). This excluded observation is then used to validate the model. However, when distance or (dis)similarity matrices are used as response variables (as in many landscape-genetic studies) and a certain population pair is selected as validation set, there may still be pairwise observations involving either of these two populations in the calibration set, which diminishes the independence of the validation set. Therefore, we propose a slightly modified LOOCV, in which all pairwise observations, involving either of the two populations in the validation set, are excluded from the calibration set (Fig. 3).

The predictive accuracy of a model is determined by comparing the value of $F_{\mathrm{ST}}$ or MAP in the "independent" validation set with that predicted by the model based on the predictor variables in the validation set. The closer the predicted $F_{\mathrm{ST}}$ or MAP value is to the true value, the better is the predictive accuracy of the model. The process of comparing true with predicted $F_{\mathrm{ST}}$ or MAP values is iterated until every population pair has been in the validation set exactly once (i.e., 97 iterations). Finally, the predictive accuracy of the model was expressed in the root mean square prediction error (RMSE), which is the root of the mean of the summed squared differences between the predicted and the true

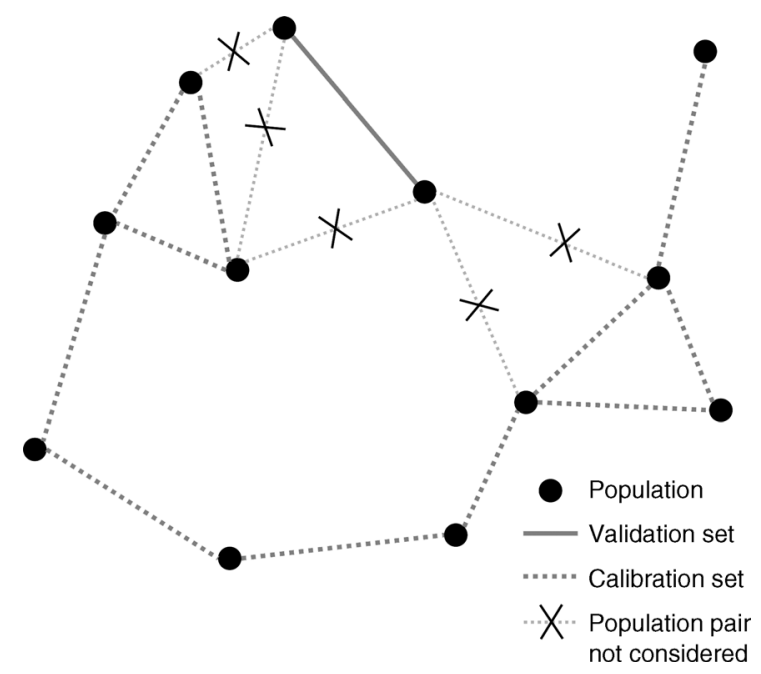

FIG. 3. An example of the splitting of all population pairs in the data set into a calibration and a validation set for leave-oneout cross-validation. As in Fig. 2, a hypothetical population network is built of links between populations that are within maximum dispersal distance. Regression models are fit to the population pairs in the calibration set and subsequently evaluated with the validation set. The validation set consists of one population pair (dark-gray line), and the calibration set consists of all other population pairs in the network (dark-gray dashed lines), excluding the population pairs that are linked to either of the two populations in the validation set (dashed lightgray lines). This process is repeated until all population pairs have been in the validation set once.

$F_{\mathrm{ST}}$ or MAP values in the validation sets (Esposito et al. 2004). Not every population occurred equally frequently in our data set, since it consisted of only those population pairs with $<3 \mathrm{~km}$ distance among populations. Therefore, the size of our calibration set fluctuated between 77 and 94 observations. For each transect width and both sets of predictor variables (i.e., with or without the MEAN-NL and MEAN-MDL), we calculated the RMSE for all possible combinations of predictor variables. The combination of predictor variables that resulted in the lowest RMSE was the model with the highest predictive accuracy. Shao (1993) concluded that model selection with LOOCV is slightly too conservative (i.e., retaining too many predictor variables), if the true model is known. In our case the true model was not known, and we therefore found this an acceptable bias. For the model with the lowest RMSE, we also calculated $R^{2}$ and the cross-validated $R^{2}$, i.e., $Q^{2}$, statistics. The latter is a measure of "goodness of prediction" (Leach 2001). We chose the model with the highest overall $Q^{2}$ to predict gene flow resulting from landscape change scenarios. All statistical analyses were performed in $\mathrm{R}$ (R Development Core Team 2012). With the "dredge" function in the R package "MuMIn" (Barton 2012), we determined all possible combinations of predictor variables from a full model. 


\section{Landscape-change scenarios and gene flow predictions}

As an illustration of how multiple regression models can be used for forecasting in landscape genetics, we predicted the effects of hypothetical landscape-change scenarios on gene flow among populations of $S$. grossum. From our data set, we selected the two population pairs that were genetically most isolated from each other and the two population pairs that were least isolated from each other. Under the assumption of isolation by distance (Wright 1943), close populations pairs have more gene flow than distant pairs, and we would simply end up selecting the two closest and two farthest population pairs. To correct for this potential effect, we calculated relative "gene flow rates" by dividing the measure of gene flow from the best-fitting model by the log of the distance (MAP/n(DISTANCE) or $F_{\mathrm{ST}} / \ln (\mathrm{DISTANCE})$, where DISTANCE is measured in meters). Between the population pairs with the highest MAP gene flow rate or lowest $F_{\mathrm{ST}}$ gene flow rate there is relatively more gene flow than would be expected from the distance between the two populations, and these populations can be considered genetically least isolated from each other. Similarly, the population pairs with the lowest MAP gene flow rate or highest $F_{\mathrm{ST}}$ gene flow rate can be considered genetically most isolated from each other. For the two genetically most isolated population pairs, landscape change was focused on increasing gene flow, while for the two genetically least isolated population pairs, we assessed whether hypothetical landscape change would negatively influence gene flow. By changing the landscape in the transects between these population pairs, we simulated four types of landscape change. In the "construction" scenario, a residential area was constructed adjacent to a small settlement. In the "restoration" scenario, an area of agricultural land was reforested as part of a river restoration project. In the "new population" scenario, some old factory buildings were demolished and a new population of $S$. grossum established itself in between the population pair under consideration. Finally, in the "rezoning" scenario, a forest plantation was converted to agricultural land.

After having implemented the above scenarios to the landscape in the transects between the four selected population pairs, we calculated new values for all predictor variables. These modified variables were entered into the model with the highest $Q^{2}$ (see Predictive models and cross-validation) to make predictions of gene flow estimates ( $F_{\mathrm{ST}}$ or MAP). We calculated the $95 \%$ prediction intervals of these new gene flow estimates using the estimated mean-squared prediction error from the LOOCV (i.e., $\mathrm{RMSE}^{2}$ ) as prediction variance. A change in gene flow was considered significant, if the observed gene flow (in the unchanged landscape) was outside the prediction interval of the predicted gene flow (in the changed landscape). Models were fitted with the "lm" function and predictions and prediction intervals were calculated with the "predict" function in R (R Development Core Team 2012).

\section{RESUlts}

Predictive model selection

From $F_{\mathrm{ST}}$ or MAP regressed against landscape metrics (i.e., $\ln$ (DISTANCE), STREAMS, HABITAT, RESIDENTIAL, AGRICULTURE, AI and COHESION), we determined the subset of predictors with the lowest RMSE (Table 1). For both response variables, the selected predictor variables were $\ln$ (DISTANCE) and AGRICULTURE for all transect widths. Nevertheless, predictor variables calculated from transects with a width of $700 \mathrm{~m}$ produced the models with the highest predictive accuracy for both response variables $\left(F_{\mathrm{ST}}, Q^{2}=0.0753\right.$; MAP, $\left.Q^{2}=0.2163\right)$. The landscape index COHESION was never selected and the index AI was only selected once, namely in the model from 100-m transects; $F_{\mathrm{ST}} \sim \mathrm{AI}+\mathrm{AGRICULTURE.}$

After adding the two population topology predictors (MEAN-MDL and MEAN-NL) to the full model, we found that the models with the highest $Q^{2}$ for both response variables were again those from transects with a width of $700 \mathrm{~m}\left(F_{\mathrm{ST}}, Q^{2}=0.1925\right.$; MAP, $Q^{2}=0.3444$; Table 1). From the differences in $Q^{2}$ value between models with and without MEAN-MDL and MEAN$\mathrm{NL}$, it was apparent that the inclusion of measures of population topology greatly improved the predictive accuracy of the models $\left(F_{\mathrm{ST}}\right.$, highest $Q^{2}$ from 0.0753 to 0.1925 ; MAP, highest $Q^{2}$ from 0.2163 to 0.3444 ; Table $1)$. For $F_{\mathrm{ST}}$ as response variable, the models with AGRICULTURE and MEAN-NL produced the lowest RMSE $(=0.0260$; transect width $=700 \mathrm{~m})$. The model MAP $\sim 0.7551-0.0265 \times \ln ($ DISTANCE $)-0.1933 \times$ RESIDENTIAL + $0.1994 \times$ AGRICULTURE -1.770 $\times 10^{-4} \times$ MEAN-MDL was selected for having the lowest RMSE $(=0.0854$; transect width $=700 \mathrm{~m})$. This model also had the highest overall $Q^{2}$ value and was therefore used in the following step to predict gene flow after landscape change. For this model, Fig. 4 shows the observed (true) MAP values plotted against predicted MAP values from leave-one-out cross-validation. Furthermore, from the models including the population topology predictors, we found that the models with MAP as response variable generally had a higher model fit than models with $F_{\mathrm{ST}}$ as response variable $\left(F_{\mathrm{ST}}, R^{2} \leq\right.$ 0.3011 ; MAP, $R^{2} \geq 0.4085$; Table 1)

\section{Prediction of gene flow}

The population pairs 13-14 and 10-34 had the highest MAP gene flow rates (0.0849 and 0.0848 , respectively), and population pairs $11-33$ and 2-21 had the lowest MAP gene flow rates (0.0182 and 0.0147 , respectively). In $700 \mathrm{~m}$ wide transects between these four population pairs (Fig. 1), we demonstrate below how landscape change may alter MAP (Fig. 5).

In the "construction" scenario between populations 13 and 14 (Fig. 5), the construction of a $0.11-\mathrm{km}^{2}$ 
TABLE 1. Results of model selection for gene flow linearly regressed against landscape metrics calculated from interpopulation transects of different widths (upper half) and later combined with measures of population topology (lower half).

\begin{tabular}{|c|c|c|c|c|c|}
\hline $\begin{array}{l}\text { Transect } \\
\text { width }\end{array}$ & $\begin{array}{l}\text { Full } / \text { selected } \\
\text { model }\end{array}$ & Model formula & RMSE & $Q^{2}$ & $R^{2}$ \\
\hline & full model & $\begin{array}{l}\text { MAP } \sim \ln (\text { DISTANCE })+\text { STREAMS + HABITAT + } \\
\quad \text { RESIDENTIAL + AGRICULTURE + AI + COHESION }\end{array}$ & & & \\
\hline 100 & selected model & $\mathrm{MAP} \sim \ln (\mathrm{DISTANCE})+\mathrm{AGRICULTURE}$ & 0.0989 & 0.1210 & 0.2513 \\
\hline 200 & selected model & MAP $\sim \ln ($ DISTANCE $)+$ AGRICULTURE & 0.0980 & 0.1362 & 0.2630 \\
\hline 400 & selected model & MAP $\sim \ln ($ DISTANCE $)+$ AGRICULTURE & 0.0965 & 0.1622 & 0.2855 \\
\hline 700 & selected model & MAP $\sim \ln ($ DISTANCE $)+$ AGRICULTURE & 0.0933 & 0.2163 & 0.3349 \\
\hline \multirow[t]{2}{*}{1000} & selected model & MAP $\sim \ln ($ DISTANCE $)+$ AGRICULTURE & 0.0947 & 0.1937 & 0.3250 \\
\hline & full model & $\begin{array}{l}F_{\mathrm{ST}} \sim \ln (\text { DISTANCE })+\text { STREAMS + HABITAT + } \\
\quad \text { RESIDENTIAL + AGRICULTURE + AI + COHESION }\end{array}$ & & & \\
\hline 100 & selected model & $F_{\mathrm{ST}} \sim \ln ($ DISTANCE $)+$ AGRICULTURE $\dagger$ & 0.0286 & 0.0229 & 0.1606 \\
\hline 200 & selected model & $F_{\mathrm{ST}} \sim \ln ($ DISTANCE $)+$ AGRICULTURE & 0.0284 & 0.0426 & 0.1736 \\
\hline 400 & selected model & $F_{\mathrm{ST}} \sim \ln ($ DISTANCE $)+$ AGRICULTURE & 0.0280 & 0.0644 & 0.1859 \\
\hline 700 & selected model & $F_{\mathrm{ST}} \sim \ln ($ DISTANCE $)+$ AGRICULTURE & 0.0279 & 0.0753 & 0.1961 \\
\hline \multirow[t]{2}{*}{1000} & selected model & $F_{\mathrm{ST}} \sim \ln ($ DISTANCE $)+$ AGRICULTURE & 0.0286 & 0.0238 & 0.1601 \\
\hline & full model & $\begin{array}{l}\text { MAP } \sim \ln (\text { DISTANCE) + STREAMS + HABITAT + } \\
\text { RESIDENTIAL + AGRICULTURE + AI + COHESION } \\
\text { + MEAN-MDL + MEAN-NL }\end{array}$ & & & \\
\hline 100 & selected model & $\begin{array}{l}\text { MAP } \sim \ln (\text { DISTANCE })+\text { RESIDENTIAL + } \\
\text { AGRICULTURE + COHESION + MEAN-MDL }\end{array}$ & 0.0866 & 0.3250 & 0.4606 \\
\hline 200 & selected model & MAP $\sim \ln ($ DISTANCE $)+$ RESIDENTIAL + MEAN-MDL & 0.0874 & 0.3129 & 0.4085 \\
\hline 400 & selected model & MAP $\sim \ln ($ DISTANCE $)+$ RESIDENTIAL + MEAN-MDL & 0.0866 & 0.3251 & 0.4170 \\
\hline 700 & selected model & $\begin{array}{l}\text { MAP } \sim \ln (\text { DISTANCE) + RESIDENTIAL + } \\
\quad \text { AGRICULTURE + MEAN-MDL }\end{array}$ & 0.0854 & 0.3444 & 0.4883 \\
\hline 1000 & $\begin{array}{l}\text { selected model } \\
\text { full model }\end{array}$ & $\begin{array}{l}\text { MAP } \sim \ln (\text { DISTANCE })+\text { AGRICULTURE + MEAN-MDL } \\
F_{\mathrm{ST}} \sim \ln (\text { DISTANCE }+ \text { STREAMS + HABITAT + } \\
\quad \text { RESIDENTIAL + AGRICULTURE + AI + COHESION } \\
\quad+\text { MEAN-MDL + MEAN-NL }\end{array}$ & 0.0869 & 0.3205 & 0.4662 \\
\hline 100 & selected model & $F_{\mathrm{ST}} \sim$ AGRICULTURE + MEAN-MDL & 0.0267 & 0.1510 & 0.2759 \\
\hline 200 & selected model & $F_{\mathrm{ST}} \sim$ AGRICULTURE + MEAN-NL $\$$ & 0.0264 & 0.1671 & 0.2875 \\
\hline 400 & selected model & $F_{\mathrm{ST}} \sim$ AGRICULTURE + MEAN-NL & 0.0261 & 0.1866 & 0.2963 \\
\hline 700 & selected model & $F_{\mathrm{ST}} \sim$ AGRICULTURE + MEAN-NL & 0.0260 & 0.1925 & 0.3011 \\
\hline 1000 & selected model & $F_{\mathrm{ST}} \sim$ AGRICULTURE + MEAN-NL $\S$ & 0.0270 & 0.1297 & 0.2641 \\
\hline
\end{tabular}

Notes: Gene flow was estimated as either pairwise mean assignment probability (MAP) or genetic differentiation $\left(F_{\mathrm{ST}}\right)$. For each transect, the DISTANCE $(\mathrm{m})$ and proportion of the landscape elements STREAMS, HABITAT, RESIDENTIAL, and AGRICULTURE $\left(\mathrm{m}^{2} / \mathrm{m}^{2}\right)$ together with the COHESION and aggregation index (AI) were calculated. Measures of population topology were calculated by averaging per population pair the number of populations within dispersal distance from either population and the mean distance (with distance originally measured in meters) to these surrounding populations (i.e., MEAN-NL and MEAN-MDL, respectively; Fig. 2). From all potential combinations of predictor variables in the full model, the model with the lowest root mean square error (RMSE) was selected for each transect width. For the selected models, estimates of goodness of prediction $\left(Q^{2}\right)$ and goodness of fit $\left(R^{2}\right)$ were calculated. The models with the lowest overall RMSE are displayed in bold for both response variables, MAP and $\mathrm{F}_{\mathrm{ST}}$.

† Same RMSE: $F_{\mathrm{ST}} \sim \mathrm{AI}+\mathrm{AGRICULTURE}$.

+ Same RMSE: $F_{\mathrm{ST}} \sim$ AGRICULTURE + AI + MEAN-NL.

$\S$ Same RMSE: $F_{\mathrm{ST}} \sim \ln ($ DISTANCE $)+$ AGRICULTURE + MEAN-MDL and FST $\sim$ AGRICULTURE + MEAN-MDL.

residential area would result in a $180 \%$ increase of the predictor RESIDENTIAL from 0.066 to $0.185 \mathrm{~m}^{2} / \mathrm{m}^{2}$ and a $17 \%$ decrease of AGRICULTURE from 0.612 to $0.509 \mathrm{~m}^{2} / \mathrm{m}^{2}$. Due to these changes, the MAP was predicted to significantly decrease from 0.566 to 0.364 . In the transect between populations 10 and 34 , we simulated a "restoration" scenario with the planting of a $0.15-\mathrm{km}^{2}$ forest patch along a stream (Fig. 5). This landscape change would bring about a $19 \%$ decrease of the predictor AGRICULTURE from 0.949 to $0.770 \mathrm{~m}^{2} /$ $\mathrm{m}^{2}$, which would result in a slight and insignificant decrease of MAP from 0.550 to 0.412. In the "new population" scenario between population 11 and 33 (Fig. 5), the demolition of old factory buildings and the establishment of a new population caused changes in RESIDENTIAL from 0.030 to $0.009 \mathrm{~m}^{2} / \mathrm{m}^{2}(70 \%$ decrease), AGRICULTURE from 0.717 to $0.737 \mathrm{~m}^{2} /$ $\mathrm{m}^{2}$ (3\% increase) and MEAN-MDL from 2072 to 1848 $\mathrm{m}$ (11\% decrease). These changes would lead to a significant increase of MAP from 0.137 to 0.373 . Lastly, in the "rezoning" scenario, a $0.27-\mathrm{km}^{2}$ piece of land was transformed from forest plantation to agriculture between populations 2 and 21 (Fig. 5). This caused a 43\% increase in AGRICULTURE from 0.296 to 0.424 $\mathrm{m}^{2} / \mathrm{m}^{2}$, and, because a forest hut would also be demolished in the process, a $3 \%$ decrease in RESIDENTIAL from 0.039 to $0.038 \mathrm{~m}^{2} / \mathrm{m}^{2}$. These changes would result in a slight and insignificant change in MAP from 0.115 to 0.162 .

\section{Discussion}

In this study, we presented how landscape-genetic models can be applied to predict the effects of landscape change on gene flow among populations of the 


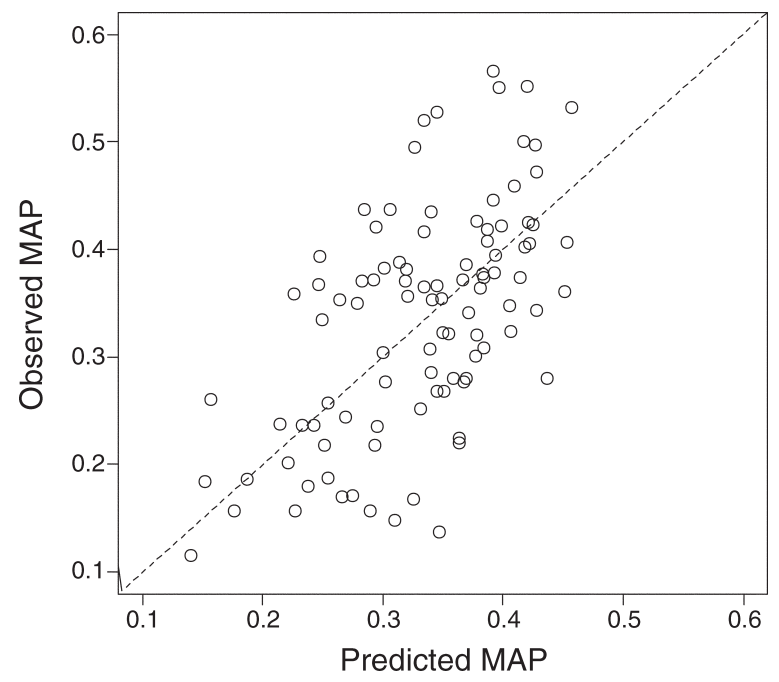

FIG. 4. Scatterplot of predicted and observed pairwise mean assignment probabilities (MAP) from a leave-one-out cross-validation of the regression model MAP $\sim \ln ($ DISTANCE) + MEAN-MDL + RESIDENTIAL + AGRICULTURE (Table 1). For an explanation of the procedure see Fig. 3. The dashed line through the origin with a slope of 1 represents the line of perfect match between predicted and observed MAP values.

vulnerable grasshopper species $S$. grossum inhabiting an intensive agricultural landscape. We showed that some landscape changes are expected to increase gene flow between less-connected population pairs, while some well-connected pairs may experience less gene flow after certain landscape changes. Species distribution modeling is widely used in species conservation to predict the effects of environmental change on the area of available habitat of certain species (Rodríguez et al. 2007). Likewise, our study opens up new possibilities for conservation practitioners to consider the effects of landscape change between patches of suitable habitat on gene flow. Furthermore, we found that the inclusion of measures of population topology strongly improved the model fit and predictive accuracy of our landscapegenetic models.

\section{Predictor variables}

Apart from the Euclidean distance among populations, we found that the proportions of agricultural land and residential areas in the transects were the most important landscape predictors for gene flow (i.e., MAP). Also working with transects and S. grossum, Keller et al. (2013a) assessed the significance of predictor variables in full landscape-genetic models, and, in their best-performing model, also found distance and the proportion of residential area to be significant predictors of MAP. However, they also found the proportion of forests, roads, and streams to be significant predictors. Instead of the proportion of forest, we decided to incorporate the proportion of agriculture. We made this choice, because we found the proportion of forest to be strongly and negatively correlated to the proportion of agriculture. Including correlated predictor variables in regression models can lead to misspecification of the sign of regression coefficients (Legendre and Legendre 1998). Whether gene flow was facilitated by agriculture or inhibited by forest or both in S. grossum cannot be determined with certainty from such landscape-genetic models (Angelone et al. 2011). A review of literature suggests both to be important. Marzelli (1995) and Griffioen (1996) mentioned that S. grossum disperses through extensive grassland and intensive agriculture, respectively. Sorens (1996) and Bonsel and Sonneck (2011) suggested high trees as a barrier to movement in S. grossum. Furthermore, we decided not to consider the proportion of roads in our study. Surprisingly, this predictor had a strong positive correlation with MAP in Keller et al. (2013a). These authors discussed that this finding may be explained by dispersal of $S$. grossum along road verges. Thus, the proportion of roads itself is most probably not facilitating gene flow, and is only an indirect predictor variable (Austin and Smith 1989). Including such predictor variables in predictive models may lead to biased forecasts. For instance, a predictive model with positive correlation between MAP and the proportion of roads would predict highest gene flow if the entire transect was paved. These issues clearly show the importance of including only "uncorrelated" causal or direct predictor variables in predictive models, as has also been emphasized for species distribution modeling (Guisan and Zimmermann 2000).

We experimented with two types of predictor variables that previously have not been used in landscapegenetic models. First, we calculated measures of habitat configuration from transects between populations. Although these predictor variables hardly added predictive accuracy to our models, we remain in favor of their use in landscape genetics. For instance, we see potential in developing landscape indices that indicate the location of certain landscape elements in a transect. Such indices would allow us to differentiate between landscape change taking place at the edge or in the middle of a transect. Furthermore, we added predictor variables that quantified population topology to our landscape-genetic models. This resulted in a substantial increase in model fit $\left(R^{2}\right)$ and predictive accuracy $\left(Q^{2}\right)$. As we expected, we found that the farther surrounding populations were from a certain population pair, the less gene flow was estimated between that population pair (i.e., negative correlation between MEAN-MDL and MAP). From this, we conclude that an important part of the gene flow between two populations is the result of indirect dispersal via surrounding populations across several generations. These findings confirm the recommendations of earlier studies (Dyer et al. 2010, Keller et al. $2013 a$ ) to more explicitly consider population topology in landscape genetics. In the current study, we included the effect of population topology (MEAN$\mathrm{MDL}$ ) as a predictor variable and only assessed its effect 


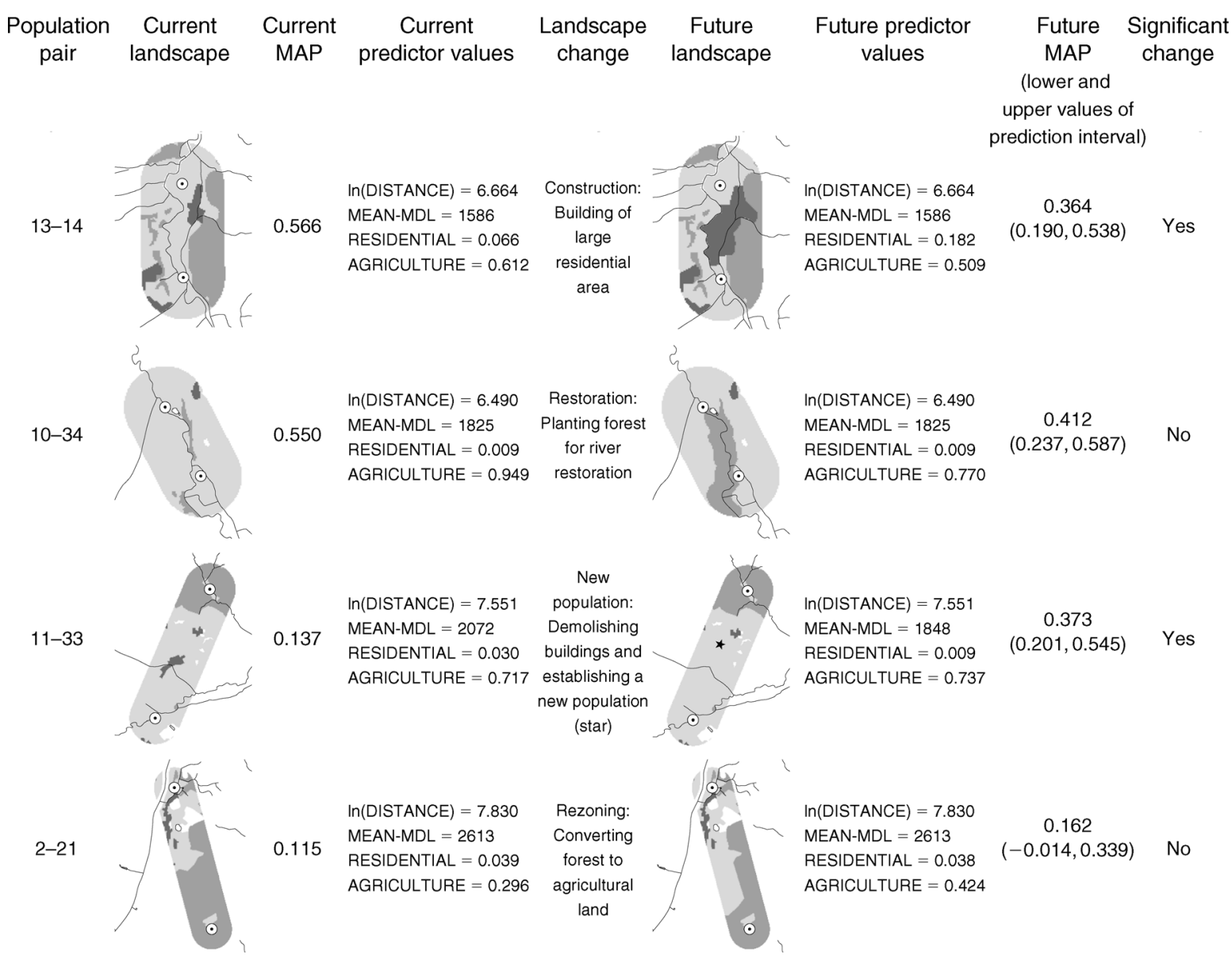

FIG. 5. The effect of hypothetical landscape-change scenarios on gene flow (pairwise mean assignment probability, MAP) between four population pairs predicted with the linear model MAP $\sim \ln ($ DISTANCE $)+$ MEAN-MDL + RESIDENTIAL + AGRICULTURE (Table 1), with all distances originally measured in meters. Four different landscape-change scenarios were implemented in the transects between the two population pairs with the highest (upper two) and lowest (lower two) gene flow rates. Between each population pair (Fig. 1), the observed MAP value, the current landscape in the transect, and the corresponding predictor variables are displayed. The hypothetical landscape change is described and applied to the landscape (future landscape) in the transect, resulting in new values of the predictor variables (future predictor values). Based on these new predictor values, future values of MAP together with the lower and upper limits of their $95 \%$ prediction intervals were predicted. Change in MAP was significant if the current value of MAP was outside the prediction intervals of the predicted MAP. Gray shading of the landscape elements are the same as in Fig. 1.

on a single population pair. In reality, however, there may be an interaction effect between population pairs, i.e., a change in gene flow between two populations will also have effects on gene flow between surrounding populations. Implementing such an effect in predictive landscape-genetic models would require more dynamic agent-based models (Landguth and Cushman 2010). Furthermore, populations located close to a certain population pair may not only enhance gene flow, but might in fact also reduce gene flow between the population pair by acting as "sponges" to dispersing individuals (Adriaensen et al. 2003).

\section{Considerations in predictive landscape genetics}

In this study we selected predictor variables based on their predictive accuracy making use of a modified leave- one-out cross-validation (LOOCV) approach. An alternative to $\mathrm{LOOCV}$ is variable selection based on AIC (Burnham and Anderson 2002). Opposed to selecting a single best model, a benefit of using AIC-based variable selection is that a set of top-ranked models is selected (e.g., Goldberg and Waits 2010, Emaresi et al. 2011), of which the regression coefficients can be averaged to define a final model. The difference in AIC value between each model and the model with the lowest AIC determines the relative weight of the models in the final model. This relative weighting of the models is biased if observations are not independent from one another (as in distance or (dis)similarity matrices [Burnham and Anderson 2002]). Goldberg and Waits (2010) propose a method to select only independent observations from dissimilarity matrices in order to 
obtain unbiased AIC differences. However, this method has been criticized (Van Strien et al. 2012), and to our knowledge no methods are currently available to adjust AIC values for nonindependent observations originating from distance or (dis)similarity matrices. Although asymptotically equivalent to AIC-based variable selection (Stone 1977, Shao 1993), we preferred LOOCV, because this intuitive approach could easily be adapted to test the predictive accuracy of our models on "independent" data. As truly independent data (i.e., using different data sets for calibration and validation) is not available in many studies, cross-validation is a commonly used alternative (i.e., using the same data set for calibration and validation [Verbyla and Litvaitis 1989]). By modifying LOOCV, models could be verified with pairwise gene flow measures originating from populations that were not used to calibrate the model. Thus, model selection with LOOCV does not make use of significance values or information criteria (e.g., AIC), but simply assesses which model best predicts gene flow between a pair of populations of which only the predictor variables are known. Nevertheless, there is an urgent need for more robust statistical techniques in landscape genetics. The nonindependence of pairwise genetic distance measures results in an unknown number of degrees of freedom (Dow et al. 1987), rendering many statistical tests or measures unsuitable for landscapegenetic analyses (e.g., Legendre and Fortin 2010). Therefore, most landscape-genetic studies draw inference simply by testing the significance of predictor variables making use of Mantel tests (Balkenhol et al. 2009b, Storfer et al. 2010), which have been specifically designed to correlate distance matrices (Mantel 1967). However, the statistical validity of Mantel tests has also recently been questioned (Guillot and Rousset 2013).

Although our models predict estimates of future gene flow, it is uncertain how fast such future gene flow values would establish in reality. With a simulation study, Landguth et al. (2010) have shown that recently established barriers to gene flow were genetically detectable within only a few generations (i.e., 1-15 generations), but that genetic patterns remained unaffected for many generations (i.e., $>100$ generations) after the removal of a barrier. We thus expect that a predicted decrease in gene flow will manifest itself in a relatively short time span, while a predicted increase may only materialize over longer time periods. To empirically confirm these expectations, landscape geneticists should focus on sampling many populations in several, replicated study areas (e.g., Short Bull et al. 2011) and resampling populations at intervals after landscape change has taken place.

We chose to use straight-line transects to quantify the landscape between populations. However, if individuals do not disperse between populations in fairly straight lines, such transects do not represent the landscape encountered by a dispersing individual (Spear et al. 2010, Van Strien et al. 2012). Contrary to straight-line transects, Van Strien et al. (2012) proposed to direct transects along dispersal routes through a specific dispersal habitat (i.e., least-cost transect analysis; LCTA). With LCTA, landscape change could, thus, not only change the composition of the landscape in the transect, but also the course of the transect as a whole. Although such changes may be ecologically realistic (i.e., species may change their dispersal route after landscape change), the complex LCTA models would need further empirical and theoretical evaluation before being implemented for predictive purposes.

Recently, Jay et al. (2012) showed how species distribution models can be used to predict the effect of climate change on the distribution of and contact zones between genetic population clusters in 20 alpine plant species. In a study area spanning across the European Alps, these authors predicted that $4^{\circ} \mathrm{C}$ warming would lead to significant changes in the genetic composition within the studied species. The study of Jay et al. (2012) presents a method to predict shifts in the genetic composition of populations, but it did not take the intervening landscape into account. On the other hand, landscape genetics focuses on the prediction of gene flow through the landscape in between populations, without taking changes in the genetic composition of the populations into account. These two aspects are closely interlinked, and combining both methods could thus add increased reliability to predictions.

\section{Different forms of population structure and specialization}

For some groups of organisms the landscape-genetic models presented in this study may not be applicable or have to be adapted. Because $S$. grossum is reproductively bound to moist areas (Baur et al. 2006), we were able to identify discrete populations of this species in the study area where moist areas were patchily distributed. For species in which individuals are more continuously distributed throughout a study area, complete sampling is impractical and the influence of the arrangement of individuals or populations on gene flow may be difficult to determine. If no spatially distinct populations are present in a focal species, genetic distance could be measured between pairs of individuals (e.g., Rousset 2000), opposed to the genetic distance measures between populations used in the current study. Alternatively, genotypes of randomly sampled individuals can be statistically grouped into genetic populations based on the principles of Hardy-Weinberg equilibrium and linkage equilibrium using assignment tests (Manel et al. 2007). Furthermore, identifying certain landscape elements that inhibit or facilitate movement may be easier for habitat specialists, such as S. grossum (Bonsel and Sonneck 2011), than for habitat generalists. The movement of habitat generalists may be less driven by the availability of certain landscape elements and more by factors like disturbance, competition for food sources, or random movement behavior. It is difficult to express these latter factors in a spatial context. For 
instance, Driezen et al. (2007) used empirical movement data from radio-tagged hedgehogs to determine the most likely relative resistance-to-movement values for certain landscape elements in their study area. They stated that their low success rate in assigning resistance values to particular landscape elements was partly due to the fact that hedgehogs are habitat generalists.

\section{Benefits for conservation planning}

With the current study, we hope to show how landscape genetic models can be applied to "real world" conservation planning. When implementing landscape change in our study area, our best predictive model could be directly applied to the conservation of $S$. grossum. Empirical studies have shown that anthropogenic landscape change often results in decreased gene flow among populations and decreased genetic variation within populations (e.g., Athrey et al. 2012). On the other hand, landscape management can also effectively reconnect isolated (meta) populations (e.g., Hale et al. 2001). Likewise, our model forecasts that some landscape changes will lead to significant decreases in gene flow between populations of S. grossum, and that gene flow can be restored between isolated populations. In situations where gene flow is predicted to decrease due to landscape change and no feasible mitigating measures exist, conservationists could resort to translocation of individuals from one population to another in order to restore genetic variation within populations (e.g., Heber et al. 2013). However, conservation scientists and practitioners should also be aware of the role that genetic isolation plays in adaptive evolution. Local adaptation of populations could be important for a species' long-term survival in a changing environment. Although gene flow may spread adaptively beneficial alleles, local adaptation can also be counteracted by gene flow (Lenormand 2002). Furthermore, gene flow between locally adapted populations may lead to "migrational meltdown" or outbreeding depression, which can reduce the fitness of populations (Crispo et al. 2011).

Despite the many scientific studies focusing on conservation and landscape genetics (Jenkins et al. 2010, Storfer et al. 2010), few conservation practitioners have started considering genetics in the management of protected areas and species (Frankham 2010, Segelbacher et al. 2010, Braunisch et al. 2012). Previous landscape-genetic studies have mainly focused on identifying gene flow facilitating or inhibiting landscape elements (Storfer et al. 2010), leaving it up to conservation practitioners to interpret and weigh this knowledge and make qualitative assessments of the severity of landscape changes on gene flow. By using cross-validated landscape-genetic models to predict gene flow changes, we produced quantitative estimates of this severity, enabling practitioners to rank potential landscape change scenarios according to their anticipated impact on gene flow. Such models not only provide a tool to avoid landscape change that can lead to severe decreases in gene flow among populations, but also assist in finding management strategies that mitigate the negative effects of inevitable landscape change (e.g., by establishing new dispersal corridors or new populations). Although we demonstrated our approach on a single species, we believe that such landscape-genetic models could be particularly useful in multispecies conservation projects. Building models for several endangered or rare species in an area and overlaying these species-specific models will allow landscape planners to select areas for development that are predicted to be least damaging to overall gene flow. With the rapid development of cheaper and faster genetic sequencing technologies (Segelbacher et al. 2010, Glenn 2011), such multispecies genetic data sets will become more widely available in the near future.

\section{ACKNOWLEDGMENTS}

We thank the Cantons of Aargau, Berne, Lucerne, and Solothurn for providing permission to sample S. grossum, Esther Jung for carrying out the genetic analysis, the Genetic Diversity Centre GDC of ETH Zurich for facilitating parts of the genetic analyses, and two anonymous referees for comments on the manuscript. This study was part of the ENHANCE project funded by the Competence Center Environment and Sustainability of the ETH Domain.

\section{Literature Cited}

Adriaensen, F., J. P. Chardon, G. De Blust, E. Swinnen, S. Villalba, H. Gulinck, and E. Matthysen. 2003. The application of 'least-cost' modelling as a functional landscape model. Landscape and Urban Planning 64:233-247.

Angelone, S., F. Kienast, and R. Holderegger. 2011. Where movement happens: scale-dependent landscape effects on genetic differentiation in the European tree frog. Ecography 34:714-722.

Athrey, G., K. R. Barr, R. F. Lance, and P. L. Leberg. 2012. Birds in space and time: genetic changes accompanying anthropogenic habitat fragmentation in the endangered black-capped vireo (Vireo atricapilla). Evolutionary Applications 5:540-552.

Austin, M. P., and T. M. Smith. 1989. A new model for the continuum concept. Vegetatio 83:35-47.

Balkenhol, N., F. Gugerli, S. A. Cushman, L. P. Waits, A. Coulon, J. W. Arntzen, R. Holderegger, and H. H. Wagner. $2009 a$. Identifying future research needs in landscape genetics: where to from here? Landscape Ecology 24:455463.

Balkenhol, N., L. P. Waits, and R. J. Dezzani. 2009b. Statistical approaches in landscape genetics: an evaluation of methods for linking landscape and genetic data. Ecography 32:818830 .

Barton, K. 2012. MuMIn: multi-model inference. R package version 1.7.7. http://CRAN.R-project.org/package=MuMIn

Baur, B., H. Baur, C. Roesti, and D. Roesti. 2006. Die Heuschrecken der Schweiz. Haupt, Bern, Switzerland.

Bennett, A. F., J. Q. Radford, and A. Haslem. 2006. Properties of land mosaics: implications for nature conservation in agricultural environments. Biological Conservation 133:250264.

Bonsel, A. B., and A.-G. Sonneck. 2011. Habitat use and dispersal characteristic by Stethophyma grossum: the role of habitat isolation and stable habitat conditions towards low dispersal. Journal of Insect Conservation 15:455-463.

Braunisch, V., R. Home, J. Pellet, and R. Arlettaz. 2012. Conservation science relevant to action: a research agenda 
identified and prioritized by practitioners. Biological Conservation 153:201-210.

Burnham, K. P., and D. R. Anderson. 2002. Model selection and multimodel inference. A practical information-theoretic approach. Springer, New York, New York, USA.

Chapman, B. B., C. Bronmark, J.-A. Nilsson, and L.-A. Hansson. 2011. The ecology and evolution of partial migration. Oikos 120:1764-1775.

Crispo, E., J. S. Moore, J. A. Lee-Yaw, S. M. Gray, and B. C. Haller. 2011. Broken barriers: human-induced changes to gene flow and introgression in animals. Bioessays 33:508518.

Dow, M. M., J. M. Cheverud, and J. S. Friedlaender. 1987. Partial correlation of distance matrices in studies of population structure. American Journal of Physical Anthropology 72:343-352.

Driezen, K., F. Adriaensen, C. Rondinini, C. P. Doncaster, and E. Matthysen. 2007. Evaluating least-cost model predictions with empirical dispersal data: a case-study using radiotracking data of hedgehogs (Erinaceus europaeus). Ecological Modelling 209:314-322.

Dyer, R. J., J. D. Nason, and R. C. Garrick. 2010. Landscape modelling of gene flow: improved power using conditional genetic distance derived from the topology of population networks. Molecular Ecology 19:3746-3759.

Emaresi, G., J. Pellet, S. Dubey, A. H. Hirzel, and L. Fumagalli. 2011. Landscape genetics of the Alpine newt (Mesotriton alpestris) inferred from a strip-based approach. Conservation Genetics 12:41-50.

Esposito, E. X., A. J. Hopfinger, J. D. Madura, and J. Bajorath. 2004. Methods for applying the quantitative structureactivity relationship paradigm. Pages 131-213 in J. Bajorath, editor. Chemoinformatics: concepts, methods, and tools for drug discovery. Humana Press, Totowa, New Jersey, USA.

Frankham, R. 2006. Genetics and landscape connectivity. Pages 72-96 in K. R. Crooks and M. Sanjayan, editors. Connectivity conservation. Cambridge University Press, New York, New York, USA.

Frankham, R. 2010. Where are we in conservation genetics and where do we need to go? Conservation Genetics 11:661-663.

Franzén, M., and S. G. Nilsson. 2007. What is the required minimum landscape size for dispersal studies? Journal of Animal Ecology 76:1224-1230.

Glenn, T. C. 2011. Field guide to next-generation DNA sequencers. Molecular Ecology Resources 11:759-769.

Goldberg, C. S., and L. P. Waits. 2010. Comparative landscape genetics of two pond-breeding amphibian species in a highly modified agricultural landscape. Molecular Ecology 19:36503663.

Goudet, J. 1995. FSTAT (version 1.2): a computer program to calculate F-statistics. Journal of Heredity 86:485-486.

Griffioen, R. 1996. Over het dispersievermogen van de Moerassprinkhaan. Nieuwsbrief Saltabel 15:39-41.

Guillot, G., and F. Rousset. 2013. Dismantling the Mantel tests. Methods in Ecology and Evolution 4:336-344.

Guisan, A., and N. E. Zimmermann. 2000. Predictive habitat distribution models in ecology. Ecological Modelling 135:147-186.

Hale, M. L., P. W. W. Lurz, M. D. F. Shirley, S. Rushton, R. M. Fuller, and K. Wolff. 2001. Impact of landscape management on the genetic structure of red squirrel populations. Science 293:2246-2248.

Hassall, C., and D. J. Thompson. 2012. Study design and markrecapture estimates of dispersal: a case study with the endangered damselfly Coenagrion mercuriale. Journal of Insect Conservation 16:111-120.

He, H., B. DeZonia, and D. Mladenoff. 2000. An aggregation index (AI) to quantify spatial patterns of landscapes. Landscape Ecology 15:591-601.

Heber, S., A. Varsani, S. Kuhn, A. Girg, B. Kempenaers, and J. Briskie. 2013. The genetic rescue of two bottlenecked South
Island robin populations using translocations of inbred donors. Proceedings of the Royal Society B 280. No. 1752. http://dx.doi.org/10.1098/rspb.2012.2228

Holderegger, R., and H. H. Wagner. 2008. Landscape genetics. BioScience 58:199-207.

Ingrisch, S. 1983. The influence of moisture on the hatching rate and the development period of the eggs in central European grasshoppers. Deutsche Entomologische Zeitschrift 30:1-15.

Jay, F., S. Manel, N. Alvarez, E. Y. Durand, W. Thuiller, R. Holderegger, P. Taberlet, and O. François. 2012. Forecasting changes in population genetic structure of alpine plants in response to global warming. Molecular Ecology 21:23542368.

Jenkins, D. G., et al. 2010. A meta-analysis of isolation by distance: relic or reference standard for landscape genetics? Ecography 33:315-320.

Keller, D., R. Holderegger, and M. J. Van Strien. $2013 a$. Spatial scale affects landscape genetic analysis of a wetland grasshopper. Molecular Ecology 22:2467-2482.

Keller, D., R. Holderegger, and M. J. Van Strien. 2013b. Data from: Spatial scale affects landscape genetic analysis of a wetland grasshopper. Dryad Digital Repository. doi:10.5061/ dryad. $17 \mathrm{~cm} 4$

Keller, D., E. Jung, and R. Holderegger. 2012a. Development of microsatellite markers for the wetland grasshopper Stethophyma grossum. Conservation Genetics Resources 4:507-509.

Keller, D., M. J. Van Strien, and R. Holderegger. 2012b. Do landscape barriers affect functional connectivity of populations of an endangered damselfly? Freshwater Biology 57:1373-1384.

Lande, R. 1998. Anthropogenic, ecological and genetic factors in extinction and conservation. Researches on Population Ecology 40:259-269.

Landguth, E. L., and S. A. Cushman. 2010. CDPOP: a spatially explicit cost distance population genetics program. Molecular Ecology Resources 10:156-161.

Landguth, E. L., S. A. Cushman, M. K. Schwartz, K. S. McKelvey, M. Murphy, and G. Luikart. 2010. Quantifying the lag time to detect barriers in landscape genetics. Molecular Ecology 19:4179-4191.

Leach, A. R. 2001. Molecular modeling: principles and applications. Pearson Education, Harlow, UK.

Legendre, P., and M. J. Fortin. 2010. Comparison of the Mantel test and alternative approaches for detecting complex multivariate relationships in the spatial analysis of genetic data. Molecular Ecology Resources 10:831-844.

Legendre, P., and L. Legendre. 1998. Numerical ecology. Elsevier, Amsterdam, The Netherlands.

Lenormand, T. 2002. Gene flow and the limits to natural selection. Trends in Ecology and Evolution 17:183-189.

Malkus, J. 1997. Habitatpraferenzen und Mobilitat der Sumpfschrecke (Stethophyma grossum L. 1758) unter besonderer Berucksichtigung der Mahd. Articulata 12:1-18.

Manel, S., F. Berthoud, E. Bellemain, M. Gaudeul, G. Luikart, J. E. Swenson, L. P. Waits, P. Taberlet. and Intrabiodiv Consortium. 2007. A new individual-based spatial approach for identifying genetic discontinuities in natural populations. Molecular Ecology 16:2031-2043.

Manel, S., M. K. Schwartz, G. Luikart, and P. Taberlet. 2003. Landscape genetics: combining landscape ecology and population genetics. Trends in Ecology and Evolution 18:189-197.

Mantel, N. 1967. Detection of disease clustering and a generalized regression approach. Cancer Research 27:209220.

Marzelli, M. 1995. Grasshopper colonisation of a restoration area, focusing on the Large Marsh Grasshopper (Mecostethus grossus). Pages 37-48 in K. M. Urbanska and $\mathrm{K}$. Grodzinska, editors. Restoration ecology in Europe. Geobotanical Institute ETH, Zurich, Switzerland. 
McGarigal, K., S. A. Cushman, and E. Ene. 2012. FRAGSTATS v4: spatial pattern analysis program for categorical and continuous maps. University of Massachusetts, Amherst, USA.

McRae, B. H. 2006. Isolation by resistance. Evolution 60:15511561.

Molinaro, A. M., R. Simon, and R. M. Pfeiffer. 2005. Prediction error estimation: a comparison of resampling methods. Bioinformatics 21:3301-3307.

Monnerat, C., P. Thorens, T. Walter, and Y. Gonseth. 2007. Rote Liste Heuschrecken. BAFU, Bern, Switzerland.

Pavlacky, D. C., A. W. Goldizen, P. J. Prentis, J. A. Nicholls, and A. J. Lowe. 2009. A landscape genetics approach for quantifying the relative influence of historic and contemporary habitat heterogeneity on the genetic connectivity of a rainforest bird. Molecular Ecology 18:2945-2960.

Piry, S., A. Alapetite, J. M. Cornuet, D. Paetkau, L. Baudouin, and A. Estoup. 2004. GENECLASS2: a software for genetic assignment and first-generation migrant detection. Journal of Heredity 95:536-539.

R Development Core Team. 2012. R: a language and environment for statistical computing. R Foundation for Statistical Computing, Vienna, Austria.

Riitters, K. H., R. V. O’Neill, C. T. Hunsaker, J. D. Wickham, D. H. Yankee, S. P. Timmins, K. B. Jones, and B. L. Jackson. 1995. A factor analysis of landscape pattern and structure metrics. Landscape Ecology 10:23-39.

Rodríguez, J. P., L. Brotons, J. Bustamante, and J. Seoane. 2007. The application of predictive modelling of species distribution to biodiversity conservation. Diversity and Distributions 13:243-251.

Rousset, F. 2000. Genetic differentiation between individuals. Journal of Evolutionary Biology 13:58-62.

SAEFL. 1998. National report of Switzerland for the convention of biological diversity. Hintermann and Weber, Montreux, Switzerland.

Schumaker, N. H. 1996. Using landscape indices to predict habitat connectivity. Ecology 77:1210-1225.

Segelbacher, G., S. A. Cushman, B. K. Epperson, M. J. Fortin, O. Francois, O. J. Hardy, R. Holderegger, P. Taberlet, L. P.
Waits, and S. Manel. 2010. Applications of landscape genetics in conservation biology: concepts and challenges. Conservation Genetics 11:375-385.

Selkoe, K. A., and R. J. Toonen. 2006. Microsatellites for ecologists: a practical guide to using and evaluating microsatellite markers. Ecology Letters 9:615-629.

Shao, J. 1993. Linear model selection by cross-validation. Journal of the American Statistical Association 88:486-494.

Short Bull, R. A., S. A. Cushman, R. Mace, T. Chilton, K. C. Kendall, E. L. Landguth, M. K. Schwartz, K. McKelvey, F. W. Allendorf, and G. Luikart. 2011. Why replication is important in landscape genetics: American black bear in the Rocky Mountains. Molecular Ecology 20:1092-1107.

Sorens, A. 1996. Zur Populationsstruktur, Mobilitat und dem Eiablageverhalten der Sumpfschrecke (Stethophyma grossum) und der Kurzflugeligen Schwertschrecke (Conocephalus doralis). Articulata 11:37-48.

Spear, S. F., N. Balkenhol, M. J. Fortin, B. H. McRae, and K. Scribner. 2010. Use of resistance surfaces for landscape genetic studies: considerations for parameterization and analysis. Molecular Ecology 19:3576-3591.

Stone, M. 1977. An asymptotic equivalence of choice of model by cross-validation and Akaike's criterion. Journal of the Royal Statistical Society Series B 39:44-47.

Storfer, A., M. A. Murphy, J. S. Evans, C. S. Goldberg, S. Robinson, S. F. Spear, R. Dezzani, E. Delmelle, L. Vierling, and L. P. Waits. 2007. Putting the 'landscape' in landscape genetics. Heredity 98:128-142.

Storfer, A., M. A. Murphy, S. F. Spear, R. Holderegger, and L. P. Waits. 2010. Landscape genetics: where are we now? Molecular Ecology 19:3496-3514.

van Strien, M. J., D. Keller, and R. Holderegger. 2012. A new analytical approach to landscape genetic modelling: leastcost transect analysis and linear mixed models. Molecular Ecology 21:4010-4023.

Verbyla, D. L., and J. A. Litvaitis. 1989. Resampling methods for evaluation classification accuracy of wildlife habitat models. Environmental Management 13:783-787.

Wright, S. 1943. Isolation by distance. Genetics 28:114-138. 